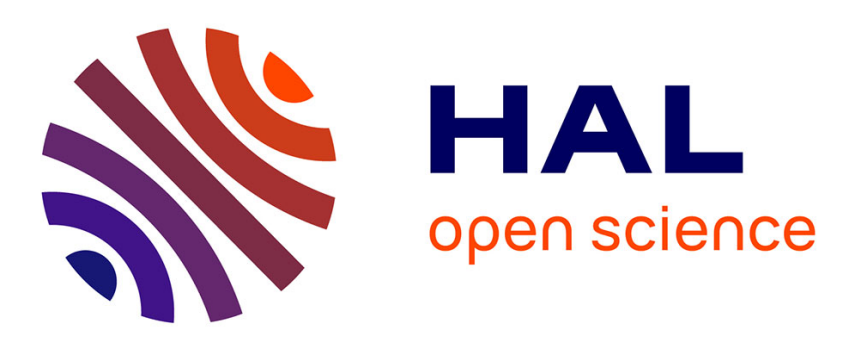

\title{
Les relations d'emploi dans la fonction publique, un défi managérial
}

Marie-Laure Buisson, Aurélie Peyrin

\section{To cite this version:}

Marie-Laure Buisson, Aurélie Peyrin. Les relations d'emploi dans la fonction publique, un défi managérial. RIMHE : Revue Interdisciplinaire Management, Homme(s) \& Entreprise, 2017, 25 (1), pp.7790. 10.3917/rimhe.025.0077 . halshs-01518973

\section{HAL Id: halshs-01518973 \\ https://shs.hal.science/halshs-01518973}

Submitted on 30 Apr 2021

HAL is a multi-disciplinary open access archive for the deposit and dissemination of scientific research documents, whether they are published or not. The documents may come from teaching and research institutions in France or abroad, or from public or private research centers.
L'archive ouverte pluridisciplinaire HAL, est destinée au dépôt et à la diffusion de documents scientifiques de niveau recherche, publiés ou non, émanant des établissements d'enseignement et de recherche français ou étrangers, des laboratoires publics ou privés. 
Auteurs invités

\section{LES RELATIONS D’EMPLOI DANS LA FONCTION PUBLIQUE, UN DÉFI MANAGÉRIAL}

Marie-Laure Buisson, Aurélie Peyrin

ARIMHE | « RIMHE : Revue Interdisciplinaire Management, Homme \& Entreprise » 2017/1 n² 25 | pages 77 à 90

ISSN 2259-2490

Article disponible en ligne à l'adresse :

http://www.cairn.info/revue-rimhe-2017-1-page-77.htm

\section{Pour citer cet article :}

Marie-Laure Buisson, Aurélie Peyrin, « Les relations d'emploi dans la fonction publique, un défi managérial ", RIMHE : Revue Interdisciplinaire Management, Homme \& Entreprise 2017/1 (n²5), p. 77-90.

DOI 10.3917/rimhe.025.0077

Distribution électronique Cairn.info pour ARIMHE.

(C) ARIMHE. Tous droits réservés pour tous pays.

La reproduction ou représentation de cet article, notamment par photocopie, n'est autorisée que dans les limites des conditions générales d'utilisation du site ou, le cas échéant, des conditions générales de la licence souscrite par votre établissement. Toute autre reproduction ou représentation, en tout ou partie, sous quelque forme et de quelque manière que ce soit, est interdite sauf accord préalable et écrit de l'éditeur, en dehors des cas prévus par la législation en vigueur en France. Il est précisé que son stockage dans une base de données est également interdit. 


\section{Auteurs invités}

\section{Les relations d'emploi dans la fonction publique, un défi managérial}

\section{Marie-Laure BUISSON ${ }^{17}$ Aurélie PEYRIN ${ }^{18}$}

En France, les réformes de la fin des années 2000, qui visaient à accroître motivation et mobilité des fonctionnaires, n'ont pas atteint leurs objectifs. Aucunes ne prenaient pas en compte la spécificité du lien d'emploi entre les fonctionnaires et l'Etat : les unes se basaient sur l'imposition d'une logique de performance importée du privé, où rien ne prouve qu'elle marche dans tous les secteurs, pour tous les postes et niveaux de compétences ;les autres sur l'affirmation idéologique qu'il fallait changer la fonction publique, car la bureaucratie serait synonyme d'apathie. Toute évocation d'une réforme d'ampleur du statut de la fonction publique renvoie d'ailleurs généralement à des positionnements idéologiques traditionnels, et conduit le plus souvent à l'extinction pure et simple du débat.

Au-delà d'un sujet clivant, se dissimule pourtant une vraie question de recherche : le statut de la fonction publique est en effet le support d'une relation d'emploi spécifique entre l'Etat et ses fonctionnaires. Peu investie par les chercheurs francophones, cette question souffre par ailleurs d'un éparpillement disciplinaire entre l'histoire, le droit, la sociologie, les sciences politiques et les sciences de gestion. Nous proposons dans cet article de contribuer à améliorer la compréhension de la relation d'emploi entre l'Etat et ses fonctionnaires en identifiant plusieurs de ses dimensions constitutives. Pour cela, nous proposons de faire dialoguer ces pans de la littérature qui ne se rencontrent jamais : les travaux (francophones) sur le statut et les réformes de la fonction publique d'une part, et les travaux (plutôt anglophones) sur la motivation de service public et la théorie du contrat implicite. Ces deux champs théoriques nous permettent d'articuler deux dimensions d'analyse du problème : une dimension institutionnelle, approchée par les caractéristiques juridiques du statut d'une part (mode de recrutement, de rémunération, d'évaluation...) et une dimension individuelle, approchée de manière plus subjective à travers les valeurs, les croyances. Dans la majorité des pays, le statut de l'emploi public s'est structuré par différenciation du droit commun, une différenciation motivée par «les missions spécifiques confiées

\footnotetext{
${ }^{17}$ Maître de Conférences, Sciences de Gestion, Aix Marseille Université, LEST (UMR-CNRS 7317) marie-laure.buisson@univ-amu.fr

${ }^{18}$ Maitre de conférences, Sociologie, Aix Marseille Université, LEST (UMR-CNRS 7317) aurelie.peyrin@univ-amu.fr
}

RIMHE - Revue Interdisciplinaire Management, Homme \& Entreprise $\mathrm{n}^{\circ} 25$ - Hiver 2017 
aux organisations publiques, conforme à l'idée du système bureaucratique au sens de Weber (Weber, 1956)» (Emery et Giauque, 2005, p.684). Mais ce référentiel idéologique s'est effondré dans le dernier quart du XXème siècle, avec une remise en question généralisée de la légitimité de l'Etat (Rosanvallon, 1992). Depuis les années 1980, de nombreux pays européens ont ainsi entrepris des réformes inspirées par le New Public Management (NPM) : mise en œuvre d'indicateurs d'activité, d'outils de gestion budgétaire et comptable, d'outils de mesure des coûts dans les organisations publiques. Les répercussions de la crise financière de 2008 sur les dettes publiques sont venues réactiver ce mouvement de rationalisation, en y ajoutant une forte contrainte à la réduction du coût de l'emploi public (Grimshaw et al., 2012). Quel est l'impact de ces réformes de l'Etat sur la gestion des personnels ? Réciproquement, quels sont les effets sur l'Etat des réformes de l'emploi public ? Plus que d'autres pays, la France est tiraillée entre injonctions institutionnelles et contraintes budgétaires d'un côté, et attachement, de l'autre, à un modèle social reposant sur une conception du service public fondé sur des principes d'égalité et d'universalité, incarné par le statut général de la fonction publique (Audier et al., 2015). Ce statut dérogatoire au droit du travail décrit et organise toujours, en France, les modes de recrutement, de rémunération, l'avancée dans la carrière et l'acquisition des droits à pension de 5,43 millions d'agents fin 2014, représentant $20 \%$ de l'emploi total ${ }^{19}$. Les relations d'emploi entre l'Etat employeur et ses agents se sont néanmoins profondément transformées en France depuis 2007, sous l'action de réformes inspirées du NPM. Que reste-t-il aujourd'hui des spécificités de l'emploi public (Emery et Giauque, 2005) ? Quelles sont enfin les nouvelles problématiques gestionnaires engendrées par ces réformes?

\section{La transformation des relations d'emploi des fonctionnaires avec l'Etat}

Deux facteurs ont justifié l'édification d'un régime dérogatoire au droit commun pour la fonction publique depuis la fin du XIXe siècle : la séparation claire entre le politique et l'administration, et le principe de recrutement assis sur les compétences (Dreyfus, 1993). Ces principes sont au cœur du statut des trois fonctions publiques françaises, unifié par un ensemble de quatre lois au milieu des années $1980^{20}$. Cependant, ce régime dérogatoire est critiqué et les réformes successives viennent bousculer le statut des fonctionnaires.

${ }^{19}$ Il s'agit des emplois qui relèvent de l'Etat (fonction publique d'Etat), de la santé (fonction publique hospitalière) et des administrations locales (fonction publique territoriale), hors emplois aidés - Source : Informations rapides, ${ }^{\circ} 303$, Insee, décembre 2015.

${ }^{20}$ Le Statut général de la fonction publique comprend quatre titres : le premier s'applique à l'ensemble des fonctionnaires (Loi n ${ }^{\circ} 83-634$ du 13 juillet 1983 portant droits et obligations des fonctionnaires) ; le second à la fonction publique de l'Etat (Loi n ${ }^{\circ} 84-16$ du 11 janvier 1984), le troisième à la fonction publique territoriale (Loi n⿳84-53 du 26 janvier 1984) et le quatrième à la fonction publique hospitalière (Loi $\mathrm{n}^{\circ} 86-33$ du 9 janvier 1986).

RIMHE - Revue Interdisciplinaire Management, Homme \& Entreprise

$\mathrm{n}^{\circ} 25$ - Hiver 2017

-78 - 


\subsection{Perte de légitimité de l'Etat et critique du statut de la fonction publique}

Dans la fonction publique, la règle et le droit prévoient précisément «les conditions d'accès (âge, diplôme, concours d'entrée), de rémunération (indices et points), de mobilité professionnelle et géographique (concours internes, mutations) de manière détaillée, ainsi que les institutions propres à les mettre en œuvre et à les contrôler (commissions paritaires)» (Reynaud et Reynaud, 1996, p. 350). Autrement dit, l'échange de travail est organisé par des «super-règles » collectivement négociées (Saglio, 2005) qui ne laissent aucune place à la négociation individuelle : après réussite du concours, un fonctionnaire est nommé par arrêté, affecté là où l'exigent les besoins du service public et selon une rémunération basée sur une grille indiciaire. En prenant cet arrêté, l'employeur public contracte une dette vis-à-vis de l'agent, auquel il s'engage à verser toute sa vie un traitement (qui augmentera progressivement avec l'ancienneté), puis, au moment de la retraite, une pension.

La justification de ce régime d'emploi dérogatoire au droit commun est socialement construite : "la spécificité des règles de droit administratif trouve sa raison d'être dans la finalité poursuivie par le service public : la recherche de l'intérêt général [...], considéré comme 'la fin ultime de l'action publique' » (Daioglou, 2009, p. 32). Mais l'édifice axiologique qui fondait la distinction public/privé vacille considérablement depuis trois décennies : la circulaire sur le renouveau du service public (1989) visait déjà la mise en place d'une gestion « plus dynamique » des personnels. L'administration n'est plus légitime de plein droit mais doit faire la preuve concrète de son efficacité : «le postulat selon lequel la gestion publique, placée au service de l'intérêt général, ne pouvait être mesurée en termes d'efficacité a fait place à l'idée que l'administration est tenue, tout comme les entreprises privées, d'améliorer sans cesse ses performances et d'abaisser ses coûts, elle est sommée de remplir ses missions, dans les meilleures conditions possibles, en veillant à la qualité de ses prestations et en utilisant au mieux les moyens à sa disposition » (Chevallier, 2003, p. 66).

Ces transformations traduisent la diffusion d'un courant idéologique qui a inspiré de nombreuses réformes dans les pays occidentaux : le nouveau management public (NPM). «Schématiquement, ces politiques regroupent des interventions gouvernementales qui prennent pour objet le système administratif et proposent d'en transformer les principales règles constitutives, historiquement mises en place de la fin du XIXe siècle au milieu du XXe siècle : relations entre administration et autorité politique, structuration de l'organisation hiérarchique, système d'emploi et modes de travail des agents publics, règles d'allocation et de gestion des ressources aux administrations ou relations entre les agents et l'administré »(Bezes, 2005, p. 431). Trois composantes majeures caractérisent les réformes liées au NPM (Rossano, Abord de Chatillon et Desmarais, 2015) : le «managérialisme », centré sur la recherche d'une meilleure maîtrise du coût de l'action publique (Kirkpatrick et al., 2005); la responsabilisation, qui renvoie à la création de structures autonomes et redevables et à

RIMHE - Revue Interdisciplinaire Management, Homme \& Entreprise

$\mathrm{n}^{\circ} 25$ - Hiver 2017 
la généralisation des pratiques de management par objectif et de reporting ; la contractualisation, qui renvoie à l'insertion des organisations publiques dans des réseaux d'acteurs multiples.

Le statut de fonctionnaire est lui aussi vivement critiqué : protection excessive du personnel, progression automatique de carrière et de salaire, fonctionnaires trop nombreux et inefficaces... Ces critiques sont principalement associées aux «privilèges spécifiques que procure un emploi public caractérisé avant tout par la sécurité de l'emploi » (Emery et Giauque, 2005, p.686). Si les critiques du statut sont aussi vivaces en France qu'ailleurs, elles n'ont pas entraîné les mêmes conséquences : alors que l'alignement sur le droit commun du travail (droit privé) concerne la majorité des agents publics en Italie ou en Allemagne, le statut des fonctionnaires français est pour l'instant réformé sans remise en cause véritable. Les historiens montrent que ces critiques sont en réalité quasiment structurelles en France : l'existence même d'un statut dérogatoire au droit commun pour les agents publics a fait l'objet de débats politiques houleux depuis le XVIIe siècle (Rouban, 2006, 2010 ; Dreyfus, 2006), et le nombre de fonctionnaires est dénoncé comme trop important depuis plus d'un siècle quel que soit leur nombre (Ruiz, 2013). Pour autant, d'importantes réformes ont radicalement changé les règles du jeu des carrières des fonctionnaires.

\subsection{Des réformes organisationnelles aux réformes statutaires : de nouvelles règles $d u$ jeu pour les fonctionnaires}

Depuis dix ans, une partie des réformes de la fonction publique française a explicitement pour but de « changer la fonction publique » (Jeannot et Rouban, 2010), c'est-à-dire de changer les règles du jeu des carrières des fonctionnaires. Si elles incarnent l'assimilation de référentiels idéologiques nouveaux, ces réformes statutaires sont aussi étroitement imbriquées dans les réformes organisationnelles, dont elles constituent le volet ressources humaines (RH). D'importantes inflexions statutaires se juxtaposent ainsi à la révision générale des politiques publiques (RGPP, 2007) et à la réorganisation territoriale de l'Etat (REATE, 2008). Entre 2008 et 2009, plusieurs textes de lois et décrets ${ }^{21}$ font évoluer les modes de recrutement et accroissent l'individualisation des modes d'évaluation et de rémunération, mais aussi les passerelles entre public et privé ou encore les possibilités de cumul.

L'un des objectifs de la RGPP était de transformer la gestion du personnel en transposant à la fonction publique les techniques de GRH du privé. La «dynamisation des parcours professionnels » par l'instauration de «droits effectifs à la mobilité »; le développement d'une «culture du résultat » et du «suivi individualisé » des agents ;

${ }^{21}$ Loi n ${ }^{\circ} 2009-972$ du 3 août 2009 «mobilité et parcours professionnels dans la fonction publique », en complément du décret $\mathrm{n}^{\circ} 2008-370$ du 18 avril 2008 sur "les conditions d'exercice des fonctions, en position d'activité, dans les administrations de l'Etat ».

RIMHE - Revue Interdisciplinaire Management, Homme \& Entreprise

$\mathrm{n}^{\circ} 25$ - Hiver 2017

-80 - 
l'ouverture et la diversification du recrutement (Voir Annexe 1) constituent trois volets de rénovation de la GRH en continuité apparente avec des processus bien engagés (Chevallier 2010). Ces nouvelles dispositions ouvrent aux fonctionnaires titulaires de nouvelles perspectives de mobilité, à travers l'accès à d'autres corps, d'autres fonctions publiques et leur apportent de nouvelles garanties, par exemple en matière de formation continue ${ }^{22}$. D'autres points de la réforme s'inscrivent plus manifestement en rupture avec les tendances antérieures: réduire le nombre de fonctionnaires, reconfigurer la fonction publique, et infléchir le statut. D'emblée, la RGPP prévoyait de diminuer le nombre de fonctionnaires de 100000 tous les trois ans, "par une refonte de l'organisation administrative et des méthodes de gestion $»^{23}$.

La réorganisation territoriale de l'Etat (REATE) est elle aussi un moment charnière pour le passage d'une «fonction publique de carrière » à une «fonction publique de métier» (Silicani 2008). La mise en place des nouvelles structures départementales interministérielles mutualise les services de l'Etat au niveau régional et départemental, en rassemblant des agents relevant de corps ministériels, ce qui renvoie à autant de règles différentes d'avancement, de mobilité, et d'attribution de primes. Surtout, avec la REATE, de nombreux agents sont par exemple confrontés à la disparition de leur emploi : certains vont pouvoir conserver le même métier au même endroit, d'autres vont devoir choisir entre leur métier et leur lieu de travail. Véritable révolution dans le droit public des fonctionnaires, la garantie d'emploi n'est désormais plus intangible : « depuis la loi du 3 août 2009, [...] le fonctionnaire sans affectation peut être placé en disponibilité d'office ou admis à la retraite lorsqu'il a manqué à ses obligations de recherche de poste ou lorsqu'il a refusé trois offres d'emploi public fermes, précises et correspondant à sa situation professionnelle » (Wolikow 2010, p. 174).

L'articulation entre réforme organisationnelle et réforme statutaire prend ici tout son sens : la loi mobilité a précisément vocation à encadrer les reconversions, les formations et attribuer des aides financières pour les déménagements. Les fusions de corps, déjà nombreuses, visent à faciliter les mobilités entre ministères, en harmonisant les règles de gestion de la carrière (et les régimes indemnitaires, souvent au cœur des " négociations » avec les représentants syndicaux). Un répertoire interministériel des métiers de l'Etat a été mis en place, pour distinguer plus nettement le poste occupé et le corps d'appartenance de l'agent. L'évaluation individuelle, les plans de formation et l'introduction de la rémunération à la performance complètent ce tableau. L'ensemble de ces changements traduit une profonde transformation du système de gestion unifiée et collective des carrières, mais aussi une délégitimation des règles statutaires et des réseaux d'acteurs constitués par les représentants du

\footnotetext{
22 La formation continue a fait l'objet d'accords-cadres en 1989, 1992 et-1996, mais la loi du 2 février 2007 consacre officiellement le droit individuel à la formation dans la fonction publique.

${ }^{23}$ Un rapport IGA-IGF-IGAS de 2012 estime à 87000 les suppressions d'emploi réalisées entre 2008 et 2010, contre 28000 les trois années précédentes.
}

RIMHE - Revue Interdisciplinaire Management, Homme \& Entreprise $\mathrm{n}^{\circ} 25$ - Hiver 2017 
personnel et les organisations syndicales, à travers les commissions administratives paritaires, consultées pour les promotions (Garabige et Lallement 2004).

Les réformes statutaires survenues depuis 2007 révèlent la spécificité des conditions d'emploi des fonctionnaires : fixées unilatéralement par l'employeur public, celui-ci peut les modifier dans l'intérêt du service sans que les agents puissent invoquer un quelconque droit acquis au maintien de règles antérieures. Ainsi, le «modèle traditionnel de socialisation par les corps relayé par des pratiques de gestion typiques des marchés internes (mobilité interne, emploi à vie, sélectivité du recrutement, fonction publique de carrière) » (Chanut et Rojot, 2011, p. 199), est progressivement transformé par l'introduction de nouveaux modes de régulation. En rompant le pacte social initial, ces évolutions pourraient perturber la nature de l'engagement des fonctionnaires. Comment ces saillies gestionnaires sont-elles perçues par les fonctionnaires? Comment gérer des fonctionnaires aux conditions d'emploi renouvelées? Les fondements de l'engagement professionnel des fonctionnaires sontils finalement modifiés?

\section{La gestion de la relation d'emploi des fonctionnaires avec l'Etat dans un contexte renouvelé}

Dans le contexte d'une inspiration croissante des logiques du secteur privé, les fonctionnaires se voient appliquer de nouvelles méthodes de gestion qui viennent questionner les fondements de leur engagement. Se pose alors la question de la gestion de ces personnels, dont les conditions d'emplois évoluent progressivement et qui « résistent » à leur façon.

\subsection{Résistance et acculturation : l'effet limité de l'importation des outils de gestion du secteur privé}

Les réformes statutaires en faveur de la mobilité, d'une «orientation métier », promeuvent des logiques managériales étrangères à la culture du service public. De façon sous-jacente, la diffusion de "mécanismes incitatifs empruntés aux entreprises du secteur privé » au sein des administrations de l'Etat devait «corriger » un modèle qui poserait problème (Alber, 2013). A première vue, «le 'retard' du secteur public en matière d'individualisation des objectifs et des rétributions n'est pourtant pas si prononcé qu'on l'entend fréquemment. » (Alber, 2013, p. 19). Surtout, une analyse détaillée révèle la capacité de résistance du modèle statutaire : tout se passe comme si l'acculturation du public aux outils du privé se faisait par détournement ou sabotage. Ainsi, «en 2006, les objectifs chiffrés étaient certes très répandus, mais finalement moins injonctifs ; les entretiens d'évaluation étaient plus généralisés mais avaient peu d'incidence effective sur les parcours et les rémunérations et les parts salariales variables, bien qu'existantes, étaient à la fois moins fréquentes et moins importantes. » (Alber, 2013, p. 19). Par ailleurs, une analyse des mêmes données distinguant les

RIMHE - Revue Interdisciplinaire Management, Homme \& Entreprise

$\mathrm{n}^{\circ} 25$ - Hiver 2017 
différents ministères met en valeur le très inégal « degré de pénétration des outils de gestion dans les administrations » (Jeannot et Guillemot, 2013, p. 100), le ministère de l'économie apparaissant comme une "figure singulière de rationalisation du travail dans l'administration » ((Alber, 2013, p. 101).

Dans de nombreux pays, la rémunération au mérite a été mise en place dans le service public et continue de s'y développer alors même que ses résultats sont très mitigés (Saint Onge et Buisson, 2012). Dans une étude portant sur les Etats-Unis, Pearce et Perry avaient en effet montré dès 1983, qu'une rémunération fondée sur les performances individuelles ne motivait pas davantage les agents de la fonction publique fédérale que leur ancien salaire à l'ancienneté. Ceci s'expliquerait notamment par un budget insuffisant pour reconnaître le mérite, une iniquité entre les catégories de personnels admissibles à la rémunération au mérite, des évaluations de la performance peu valides, une faible variance dans les augmentations au mérite octroyées, etc. (Saint Onge et Buisson, 2012). L'enquête de Marsden et Richardson (1994) en Angleterre révèle que la rémunération au mérite n'a pas non plus les effets attendus sur la motivation des agents dans un centre des impôts. Comme les primes liées à la performance, ces incitations monétaires seraient donc inefficaces dans le secteur public parce qu'elles entrent en contradiction avec les valeurs des agents et les registres d'engagement professionnels qu'ils mobilisent (Bacache-Beauvallet 2006).

C'est au nom de ces valeurs spécifiques que s'élèvent des critiques de plus en plus nombreuses contre le modèle et la philosophie du NPM, et contre les pratiques managériales qu'il a favorisées: «perte de finalité de l'action des organisations publiques au profit de dimensions purement économiques et financières » (Chanut, Chomienne et Desmarais, 2015, p.235), avec des conséquences négatives quant à la qualité du service au public (Diefenbach, 2009) et aux conditions de travail (Abord de Chatillon et Desmarais, 2012). Il s'agit alors de questionner l'usage d'approches gestionnaires pour résoudre des problèmes de nature plutôt politique (Bao et al., 2013). Ainsi, la recherche d'efficience ne serait pas le principal critère de légitimité de l'action publique et la question des valeurs et de la motivation de service public reste entière.

\subsection{La motivation de service public en question}

Le concept de motivation à l'égard du service public (MSP) interroge les fondements de la motivation des personnels fonctionnaires, dans une conception centrée sur les valeurs (Perry, 1996 ; Desmarais, Edey-Gamassou, 2014). Initialement développée par Perry et Wise (1990), la MSP correspond à « la prédisposition à répondre à des motifs propres principalement ou uniquement aux institutions publiques ». Vandenabeele (2007, p.547) en propose la définition suivante : « [la motivation de service public consiste en] une croyance, des valeurs et des attitudes qui se situent au-delà de l'intérêt personnel, concernent une entité politique plus large et motive les individus à agir de

RIMHE - Revue Interdisciplinaire Management, Homme \& Entreprise

$\mathrm{n}^{\circ} 25$ - Hiver 2017

-83 - 
manière appropriée ». Ce concept met l'accent sur «l'attitude, initialement observée au sein de la haute fonction publique, qui consiste à s'impliquer au service de l'intérêt général » (Desmarais et Edey-Gamassou, 2014, p.134).

Les recherches se regroupent principalement autour de deux questions (Belle et Ongaro, 2014): la conceptualisation et la mesure de la MSP, et l'analyse de ses antécédents et conséquences. Quatre déterminants de la MSP sont, par exemple, identifiés chez les fonctionnaires tunisiens : la sécurité de l'emploi, le prestige de la fonction publique, le service de l'intérêt général ainsi que le patriotisme et le sens du devoir. Ce serait ainsi, « la promesse d'une carrière à vie et la sécurité matérielle [qui] galvanisent, toutes proportions gardées, les gestionnaires publics » (Dahmani, 2015, p.142). Les déterminants de la MSP sont « intrinsèquement en phase avec l'éthique du public et négativement corrélés aux récompenses matérielles »(Dahmani, 2015, p.134).

Les résultats de ces travaux convergent pour montrer que les dispositifs de gestion mis en place valorisent les récompenses extrinsèques, alors que la plupart des agents sont animés d'une motivation intrinsèque (Perry et Wise, 1990 ; Brewer, 2008). Ainsi, se concentrer sur des récompenses extrinsèques comme des primes à la performance pourrait être contre-productif, voire même mener à une moindre productivité si les motivations sont clairement orientées service (Deci, 1971 ; Crewson, 1997). Reprenant les travaux de Deci et Ryan (1985), Emery souligne cependant que les récompenses extrinsèques peuvent renforcer la motivation intrinsèque sous certaines conditions, notamment «lorsqu'elles sont perçues comme feedbacks formatifs, renforçant le sentiment d'autodétermination » (Emery, 2012, p. 495).

Cependant, si les antécédents de la MSP sont désormais largement étudiés, rares sont les travaux qui analysent véritablement l'impact des dispositifs de gestion et des pratiques de management sur la motivation des fonctionnaires (Emery et Giauque, 2012). De fait, « la motivation des agents publics gagnerait à s'appuyer sur un cadre conceptuel plus riche qui reflète la complexité de l'univers post-bureaucratique au sein duquel ils évoluent actuellement»(Emery, 2012, p.493). Que reste-t-il en effet de la MSP lorsque les réformes contribuent à transformer le contrat social historique entre l'Etat et les fonctionnaires?

\subsection{L'apport de la théorie du contrat psychologique}

Pour explorer « la place et l'engagement professionnel des agents publics dans l'aprèsfonctionnariat » (Desmarais, 2013, p.61), nous proposons de mobiliser la théorie du contrat psychologique. Celle-ci s'intéresse à la relation employeur/employé et aux obligations respectives des deux parties prenantes concernant la relation d'emploi, notamment dans sa partie informelle. Comme le soulignent De Rozario et Jardat (2011, p.95), « la relation d'emploi est d'abord un contrat psychologique ». Le contrat psychologique renvoie à la «croyance d'un individu relative aux termes et aux

RIMHE - Revue Interdisciplinaire Management, Homme \& Entreprise

$\mathrm{n}^{\circ} 25$ - Hiver 2017 
conditions d'un accord d'échange réciproque entre cette personne cible et une autre partie. Les questions clés ont trait à la croyance qu'une promesse a été faite et une compensation offerte en échange, liant les parties à un ensemble d'obligations réciproques » (Rousseau, 1989, p.123). Cette définition met en exergue le caractère informel et interprétatif du contrat psychologique : "un individu perçoit, en effet, de manière tout à fait individuelle et subjective les promesses qui lui sont faites par l'autre partie. De la même façon, c'est l'individu et lui seul qui perçoit la réalisation de ces promesses, c'est-à-dire leur respect, leur dépassement ou leur rupture » (MullenbachServayre, 2009, p. 3). Lorsque le salarié a, par exemple, la perception d'un écart entre les promesses de son employeur et les rétributions effectivement reçues, il considère le contrat psychologique comme rompu. Les effets sur les attitudes et comportements au travail d'une telle rupture perçue du contrat ont été largement étudiés dans le secteur privé : baisse de l'implication, de la loyauté, de la confiance, hausse de l'intention de quitter, etc. (Guerrero, 2003 ; Mullenbach-Servayre, 2009).

Un contrat psychologique spécifique au secteur public semble identifiable : le contrat relationnel, « centré sur la stabilité de la relation d'emploi, le long terme, la carrière et la confiance mutuelle, [qui] correspond à la relation d'emploi traditionnelle dans le secteur public» (Rossano et al., 2015, p.60). Or, les nouvelles pratiques organisationnelles introduites par le NPM dans les organisations publiques feraient au contraire plutôt référence au contrat transactionnel, défini par une « relation de court terme et basé sur un salaire dépendant des performances » (Rossano et al., 2015, p.60). Comment définir alors le «nouveau » contrat psychologique entre fonctionnaires et Etat employeur ? Comment se décline-t-il selon les catégories de personnel ? Les réformes et différentes pratiques du NPM ne se traduisent-elles pas pour les fonctionnaires par une rupture du contrat psychologique, voire, selon certains théoriciens (Mullenbach-Servayre, 2009), par sa «violation»? D'une façon plus indirecte, quel est l'impact de l'introduction de contrats à durée déterminée sur le ou les contrats psychologiques noués entre les fonctionnaires et l'Etat dans le secteur public ?

\section{Conclusion : La motivation de service public peut-elle perdurer si le contrat psychologique entre les fonctionnaires et l'Etat est rompu ?}

Les relations d'emploi qui lient l'Etat employeur et ses agents, notamment les fonctionnaires, ont été considérablement bousculées par les réformes survenues dans les fonctions publiques françaises depuis 2007. Nouvelles pratiques de gestion destinées à faciliter la mobilité dans un contexte de réorganisation des structures de l'Etat, outils d'individualisation des relations salariales inspirés du secteur privé : ces changements sont susceptibles de bouleverser les fondements de l'engagement professionnel des agents publics. Plusieurs études soulignent la difficile appropriation de ces nouvelles pratiques gestionnaires. Elles incitent à s'interroger sur de nouvelles formes de management à inventer pour relever le défi de préserver la mobilisation de

RIMHE - Revue Interdisciplinaire Management, Homme \& Entreprise

$\mathrm{n}^{\circ} 25$ - Hiver 2017 
ces personnels au statut d'emploi renouvelé. Si les contractuels continuent à coexister de façon durable avec les fonctionnaires, quels outils mettre en place pour gérer des personnels aux statuts d'emploi différenciés? Les recherches sur la motivation de service publique et sur le contrat psychologique sont susceptibles de les aider à négocier au mieux l'élaboration du management public de demain.

Notre article plaide également pour une approche multidimensionnelle et pluridisciplinaire de la relation d'emploi. Face au bouleversement des règles du jeu organisant les carrières dans la fonction publique, dans un contexte de réorganisations contraintes par la maîtrise du déficit budgétaire, on ne peut pas se cantonner à une vision juridique ou comptable des changements: il est essentiel de prendre en considération le contrat implicite noué entre chaque fonctionnaire et l'Etat.

Les données statistiques publiées dans les rapports annuels sur l'état de la fonction publique indiquent la montée en qualification des nouveaux entrants et l'accroissement de l'âge moyen à l'entrée dans la fonction publique. S'il est fort probable que les attentes et les représentations de ces nouvelles générations de fonctionnaires seront différentes de celles de leurs ainés, il est loin d'être assuré que la nature relationnelle de leur contrat psychologique disparaisse. Le défi à relever pour le management public n'est-il pas dès lors de l'intégrer dans ses nouvelles pratiques?

\section{Références bibliographiques}

Abord De Chatillon E., Desmarais C. (2012), Le nouveau management public est-il pathogène ?, Management International, vol.16, $\mathrm{n}^{\circ} 3$, p. 10-24.

Alber A. (2013), Management et nouvelle gestion publique : limites et paradoxes de l'imitation du privé, La nouvelle revue du travail, $\mathrm{n}^{\circ} 2$, p. 1-28.

Audier F., Bacache-Beauvallet M., Courtioux P., Gautie J. (2015), Politique salariale et mode de rémunération dans la fonction publique en France depuis le début des années 2000, Revue Française d'Administration Publique, p. 213-229.

Bacache-Beauvallet M. (2006), Les limites de l'usage des primes à la performance dans la fonction publique, Document de travail, EDHEC.

Bao G., Wang X., Larsen G.L., Morgan D.F. (2013), Beyond New Public Governance: a value-based global framework for performance management, governance, and leadership, Administration and Society, vol.45, n 4, p. 443-467.

Belle N., Ongaro E. (2014), Le NMP, les réformes administratives et la motivation au service public : améliorer le dialogue entre les agendas de recherche, Revue Internationale des Sciences Administratives, vol. 80, p. 397-416.

Bezes P. (2005), Le modèle de «l'Etat stratège » : genèse d'une forme organisationnelle dans l'administration française, Sociologie du travail, vol. $47, \mathrm{n}^{\circ} 4$, p. 431-450. 
Bezes P. (2010), Morphologie de la Révision générale des politiques publiques. Une mise en perspective historique et comparative, Revue française d'administration publique, $\mathrm{n}^{\circ} 4$, p. 769-796.

Brewer G.A. (2008), Employee and organizational performance, in Perry J.L., Hondeghem A., Eds, Motivation in Public Management: the call of public service, Oxford, Oxford University Press, p. 136-156.

Chanut V., Chomienne H., Desmarais, C. (2015), Pratiques valorielles et management public, Revue Internationale des Sciences Administratives, vol.81, p.235-242.

Chanut V., Rojot J. (2011), Réinventer l'évaluation. Les nouvelles règles du jeu RH dans une administration publique, Revue Management \& Avenir, vol. 9, $\mathrm{n}^{\circ} 49$, p. 195222.

Chevallier J. (2003), L'Etat post-moderne, Paris, LGDJ.

Chevallier J. (2010), Révision générale des politiques publiques et gestion des ressources humaines, Revue Française d'Administration Publique, n 136, p. 907-918. Crewson P.E. (1997), Public service motivation: building empirical evidence of incidence and effect, Journal of Public Administration Research and Theory, $\mathrm{n}^{\circ} 4$, p.499-518.

Daioglou H. (2009), La gestion de l'emploi précaire dans la fonction publique. Vers une logique d'emploi privé, Marseille, Presses universitaires d'Aix-Marseille.

Dahmani A. (2015), La motivation de service public des gestionnaires publics tunisiens à l'aune de la révolution. Vers un contrat psychologique renouvelé?, Revue Française de Gestion, vol.6, n²51, p. 131-147.

Deci E.L. Ryan R.M. (1985), Intrinsic motivation and self-determination in human behavior, New York, Plenum Press.

Deci E.L. (1971), Effects of externally mediated Rewards on intrinsic motivation, Journal of Personality and Social Psychology, ${ }^{\circ} 18$, p.105-115.

De Rozario P., Jardat R. (2011), Psychological contracts in organizations, Annales des Mines - Gérer \& Comprendre, vol.4, $\mathrm{n}^{\circ} 106$, p.95-97.

Desmarais C. (2013), Recension d'ouvrage, Gestion et Management Public, vol.1, n³, p. 60-63.

Desmarais C., Edey-Gamassou C. (2014), All motivated by public service? The links between hierarchical position and public service motivation, Revue Internationale des sciences administratives / international review of administrative science, vol.80, $\mathrm{n}^{\circ} 1$, p. 133-152.

Diefenbach T. (2009), New Public management in public sector organizations: the dark sides of managerialistic "enlightenment", Public Administration, vol.87, n4, p.892-909.

Dreyfus F. (1993), Les fondements philosophiques et politiques des systèmes de fonction publique. Les cas français et britannique, Revue du droit public, $\mathrm{n}^{\circ} 1, \mathrm{p} .57-74$. Dreyfus F. (2006), Servir l'Etat, un idéal encore moderne ?, Pouvoirs, n 117, p. 5-15.

RIMHE - Revue Interdisciplinaire Management, Homme \& Entreprise

$\mathrm{n}^{\circ} 25$ - Hiver 2017 
Emery Y., Giauque D. (2005), Emploi dans les secteurs public et privé : vers un processus confus d'hybridation, Revue Internationale des Sciences Administratives, vol.71, n 4 , p. 681-699.

Emery Y. (2012), La diversité des motivations des employés publics. Recherche exploratoire dans un contexte post-bureaucratique en Suisse, Revue française d'administration publique, vol.2, $\mathrm{n}^{\circ} 142$, p. 491-515.

Emery Y., Giauque D. (2012), Motivations et valeurs des agents publics à l'épreuve des réformes, Laval, Presses de l'Université de Laval.

Garabige A., Lallement M. (2004), L'action collective dans la fonction publique : un angle mort de la sociologie des relations professionnelles ?, Sociologia del Lavoro, $\mathrm{n}^{\circ} 95$, p. $128-143$.

Guerrero S. (2003), Le respect des promesses perçues par les salariés : contribution à l'audit du départ volontaire, Actes de la 5e université de printemps de l'audit social, IAE de Corse, 22-23 et 24 mai, p. 200-208.

Grimshaw D., Rubery J., Marino S. (2012), Public sector pay and procurement in Europe during the crisis: The challenges facing local government and the prospects for segmentation, inequalities and social dialoguei, Brussels, European Commission.

Jeannot G., Guillemot D. (2013), Modernisation et bureaucratie, l'administration d'Etat à l'aune du privé, Revue Française de sociologie, n ${ }^{\circ}$ 54, p. 83-110.

Jeannot G., Rouban L. (2010), Changer la fonction publique, Revue Française d'Administration Publique, ${ }^{\circ} 132$, p. 665-672.

Kirkpatrick I., Ackroyd S., Walker R. (2005), The new managerialism and public service professions, Basingstoke, Palgrave.

Marsden D., Richardson R. (1994), Performing for pay? The effects of "merit pay" on motivation in a public service, British Journal of Industrial Relations, vol.32, $\mathrm{n}^{\circ} 2$, p.243-261.

Mullenbach-Servayre A. (2009), Le contrat psychologique : un levier du comportement au travail ?, Revue de Gestion des Ressources Humaines, vol.2, n ${ }^{\circ} 2$, p.2-12.

Pearce J. L., Perry J. L. (1983), Federal merit pay: a longitudinal analysis, Public Administration Review, vol.43, n⿳⺈, p. 315-325.

Perry J. L. (1996), Measuring public service motivation: an assessment of construct reliability and validity, Journal of Public Administration Research and Theory, vol. 6, $\mathrm{n}^{\circ} 1$, p. $5-22$.

Perry J. L., Wise L.R. (1990), The motivational bases of public service, Public Administration Review, vol.50, n³, p. 367-373.

Reynaud E., Reynaud J-D. (1996), La régulation des marchés internes du travail, Revue française de sociologie, vol. 37, $\mathrm{n}^{\circ}$ 3, p. 337-368.

Rossano M., Abord De Chatillon E., Desmarais C. (2015), Rupture du contrat psychologique et risques psychosociaux : une recherche intervention dans le cadre de

RIMHE - Revue Interdisciplinaire Management, Homme \& Entreprise

$\mathrm{n}^{\circ} 25$ - Hiver 2017 
la théorie de la conservation des ressources, Revue de gestion des ressources humaines, vol.1, $\mathrm{n}^{\circ} 95$, p. 58-77.

Rouban L. (2006), Réformer l'Etat : politique de l'emploi public et modèle social, Revue Administrative, $\mathrm{n}^{\circ} 354$, p. 638-648.

Rouban L. (2010), Réorganiser ou changer l'Etat ?, Regards sur l'actualité, n³60, p. 17-22.

Rousseau D. M. (1989), Psychological and implied contracts in organizations, Employee responsibilities and Rights Journal, vol.2, n², p. 121-139.

Ruiz E. (2013), Trop de fonctionnaires? Contribution à une histoire de l'Etat par ses effectifs (France, 1850-1950), Thèse pour le doctorat d'histoire, EHESS.

Saglio J. (2005), Le droit à la carrière des officiers de la marine, Revue Française d'Administration Publique, $\mathrm{n}^{\circ}$ 116, p. 639-650.

Saint-Onge S., Buisson M-L. (2012), La rémunération au mérite dans le secteur public : bilan des connaissances et avenues de recherche, Management International, vol.16, $\mathrm{n}^{\circ} 3$, p. 75-91.

Silicani J-L. (2008), Livre blanc sur l'avenir de la fonction publique, rapport au ministre du budget, des comptes publics et de la fonction publique.

Vandenabeele W. (2007), Toward a public administration theory of public service motivation, Public Management Review, vol.9, n 4, p. 545-556.

Wolikow J. (2010), Fonctionnaires et salariés : différences, convergences, L'Actualité juridique. Fonctions publiques, $\mathrm{n}^{\circ}$ 4, p. 172-179. 


\section{Annexe 1 \\ Trois aspects des réformes statutaires : mobilité, formation, concours}

La loi mobilité (2009) facilite et étend les règles de mise à disposition (au sein ou entre les 3FP, dans les organisations internationales et les Etats étrangers). Elle rend possible la mise à disposition de salariés de droit privé dans les administrations d'Etat, et réciproquement. Elle assouplit aussi les règles relatives au cumul d'activité. Le décret 2010-1402 du 12 novembre 2010 relatif à la situation de « réorientation professionnelle » introduit d'autres dispositions importantes : tous les corps et cadres d'emploi sont désormais accessibles aux fonctionnaires par détachement ou intégration directe (l'administration ne peut s'y opposer qu'en raison de nécessité de service ou d'un avis d'incompatibilité / commission déontologie). Il introduit des dispositions financières pour faciliter la mobilité imposée au fonctionnaire en cas de restructuration.

D'autres changements réglementaires visent à «inciter les agents publics à améliorer en permanence la qualité de leurs prestations », en instaurant " une meilleure évaluation des performances des intéressés [qui] doit conduire à une différenciation des trajectoires professionnelles en fonction des résultats obtenus », en contradiction avec la culture de l'égalitarisme. Entre 2002 et 2009, plusieurs décrets ont institutionnalisé ces dispositifs d'évaluation et rénové les procédures de notation. S'y ajoutent des modalités préexistantes, qu'il s'agit d'utiliser différemment : réductions ou majorations d'ancienneté, attribution des primes, modulation des rémunérations et prime de performance en catégorie A (décrets du 11/08/2006 et décret du 22/12/2008).

Par ailleurs, la loi du 2 février 2007 vise à adapter les concours pour que la FP représente mieux la société, tout en prenant en compte les compétences plus largement que les qualifications. Cela implique d'assouplir les critères de recrutement : ouvrir l'accès de la fonction publique aux ressortissants de l'Union européenne, envisager la pérennisation des contractuels, alléger le contenu des concours et prendre en compte l'expérience professionnelle. Certaines mesures visent spécifiquement la diversification de la FP : la charte pour la promotion de l'égalité du $1^{\mathrm{er}}$ décembre 2008 prévoit la formation des membres des jurys de recrutement, la mise en place de classes préparatoires intégrées et d'allocations pour la diversité dans la fonction publique. La loi du 3 août 2009 supprime enfin toutes les limites d'âge aux concours de recrutement. 